IMA Journal of Mathematical Control and Information (2015) Page 1 of 18

doi:10.1093/imamci/dnv022

\title{
On the robustness of linear and non-linear fractional-order systems with non-linear uncertain parameters
}

\author{
IBRAHIMA N'DOYE* \\ Computer, Electrical and Mathematical Sciences and Engineering Division (CEMSE), \\ King Abdullah University of Science and Technology (KAUST), Thuwal 23955-6900, \\ Kingdom of Saudi Arabia \\ *Corresponding author: ibrahima.ndoye@kaust.edu.saｉbndoye.cran@gmail.com \\ MoHAmed DaROUACH \\ Research Center for Automatic Control of Nancy (CRAN UMR, 7039, CNRS), \\ University of Lorraine, IUT de Longwy, 186 rue de Lorraine, \\ 54400 Cosnes et Romain, France \\ Holger Voos \\ Faculty of Science, Technology and Communication (FSTC), University of Luxembourg, 6, \\ rue Richard Coudenhove-Kalergi, Luxembourg L-1359, Luxembourg
}

AND

Michel ZASADZINSKI

Research Center for Automatic Control of Nancy (CRAN UMR, 7039, CNRS), University of Lorraine, IUT de Longwy, 186 rue de Lorraine, 54400 Cosnes et Romain, France

[Received on 21 June 2013; revised on 9 November 2014; accepted on 26 April 2015]

\begin{abstract}
This paper presents the robust stabilization problem of linear and non-linear fractional-order systems with non-linear uncertain parameters. The uncertainty in the model appears in the form of the combination of 'additive perturbation' and 'multiplicative perturbation'. Sufficient conditions for the robust asymptotical stabilization of linear fractional-order systems are presented in terms of linear matrix inequalities (LMIs) with the fractional-order $0<\alpha<1$. Sufficient conditions for the robust asymptotical stabilization of non-linear fractional-order systems are then derived using a generalization of the Gronwall-Bellman approach. Finally, a numerical example is given to illustrate the effectiveness of the proposed results.
\end{abstract}

Keywords: linear and non-linear fractional-order systems; linear matrix inequality (LMI); generalization of Gronwall-Bellman lemma; robust stabilization; parameter uncertainties.

\section{Introduction}

The question of stability is crucial in control theory. In the field of fractional-order control systems, there are many challenging and unsolved problems related to stability theory, with robust stability, bounded input-bounded output stability, and internal stability to name a few. Results on the stability of fractional-order control systems have been presented in Matignon (1996), Chen et al. (2006) and Petrás et al. (2004a). The issue of stability for fractional-order linear time-invariant (FO-LTI) systems with interval uncertainties, the stability issue has been addressed first in Petrás̆ et al. (2004a) and then further detailed in Chen et al. (2006), Petráš et al. (2004b) and Ahn et al. (2007), even 
with fractional-order interval uncertainties. Recently, a necessary and sufficient condition to evaluate non-commensurate fractional-order systems bounded input, bounded output stability has been presented in Sabatier et al. (2013).

For uncertain fractional-order systems with interval coefficients described in state-space form, the robust stability problem has been solved in Chen et al. (2006), where the matrix perturbation theory was used to find the range of the corresponding interval eigenvalues. The Lyapunov inequality was used in Ahn et al. (2007) to reduce the conservatism in the robust stability test of interval uncertain (FO-LTI) systems. It is shown in Ahn et al. (2007) that the robust stability of some specific (FO-LTI) systems with commensurate orders $\alpha, 1 \leqslant \alpha<2$, can be effectively checked, regardless of the interval perturbation magnitudes. In Xing and Lu (2009), sufficient conditions for the robust stability and stabilization of linear fractional-order systems with non-linear uncertain parameters based on a LMI approach with the fractional-order $\alpha$ belonging to $1 \leqslant \alpha<2$ have been presented. Recently, a novel fractional-order sliding mode controller for robust stabilization and synchronization problems of a class of fractional-order chaotic systems in the presence of model uncertainties and external disturbances has been investigated using the fractional-order version of the Lyapunov stability theory by Aghababa (2012).

In Lu and Chen (2010), sufficient conditions for the robust stability and stabilization of linear fractional-order interval systems with the fractional-order $\alpha$, where $0<\alpha<1$, have been investigated. This paper derived the results of N'Doye et al. (2010) to establish sufficient conditions for nonlinear fractional-order systems with $0<\alpha<1$ using LMI formulation and a new generalization of the Gronwall-Bellman.

In this paper, sufficient conditions for the robust stabilization of linear and non-linear fractionalorder systems with non-linear uncertainty parameters with fractional commensurate orders $\alpha, 0<\alpha<1$, are presented in terms of linear matrix inequalities (LMIs) in the linear case and using the generalization of the Gronwall-Bellman lemma in the non-linear case.

Another approach to stabilize non-linear systems is based on the Gronwall-Bellman lemma (see Pachpatte, 1973; Desoer and Vidyasagar, 1975). For example, this lemma has been applied to the exponential stability of non-linear affine systems by Zevin and Pinsky (2003), non-linear observer synthesis by Shimizu (2000), robust stabilization and observation of non-linear uncertain systems by Żak (1990), and robust stability of linear systems by Chen and Wong (1987) and Jetto and Orsini (2007). The generalization of the Gronwall-Bellman inequality provides an explicit bound to the unknown function and has been a powerful tool in the study of quantitative properties and stability of solutions of fractional-order equations (see N'Doye et al., 2009a,b, 2011). Based on the generalization of the Gronwall-Bellman inequality, we aim to establish a set of simple norm criteria of the robust stability for the non-linear fractional-order systems.

In Section 2, we provide some background on the fractional derivative and the two-parameter Mittag-Leffler function, which plays a very important role in fractional calculus. In Section 3, a sufficient and necessary condition for asymptotical stability of fractional-order systems with fractional-order $\alpha$ belonging to $0<\alpha<1$ is presented. In Section 4, uncertain non-linear fractional-order systems are presented. In Sections 5 and 6, the important issues related to linear and non-linear fractional-order systems are studied, respectively. Sufficient conditions for the robust stabilization of such linear and non-linear fractional-order systems are presented in terms of LMIs and using the generalization of the Gronwall-Bellman lemma.

Notations. $\|x\|=\sqrt{x^{\mathrm{T}} x}$ and $\|A\|=\sqrt{\lambda_{\max }\left(A^{\mathrm{T}} A\right)}$ are the Euclidean vector norm and the spectral matrix norm, respectively, where $\lambda_{\max }\left(A^{\mathrm{T}} A\right)$ is the maximal eigenvalue of the symmetric matrix $A^{\mathrm{T}} A .(f(\cdot))_{i}$ stands for the $i$ th component of vector field $f(\cdot), M^{\mathrm{T}}$ is the transpose of $M \cdot \operatorname{Sym}\{X\}$ is used to denoted $X^{\mathrm{T}}+X . \otimes$ stands for the Kronecker product. 


\section{Preliminaries}

\subsection{Definition of the fractional derivatives}

Fractional-order integration and differentiation are the generalization of their integer-order counterparts. Efforts to extend the specific definitions of the traditional integer order to the more general arbitrary order context led to different definitions for fractional derivatives (Das, 2008). One of the basic functions of fractional calculus is Euler's Gamma function which is defined by the integral

$$
\Gamma(z)=\int_{0}^{\infty} e^{-t} t^{z-1} \mathrm{~d} t
$$

which converges in the right half of the complex plane, i.e. $\operatorname{Re}(z)>0$. The correct definition of fractional derivative initial conditions needs to take into account the states of integer order integrators and more particularly the distributed state of the fractional integrator, we refer the readers to Trigeassou and Maamri $(2010,2011)$ and Trigeassou et al. $(2011,2013)$ for more details. One of the limitations of the definitions of fractional-order differentiation and model representations is the absence of initial memory effect. In view of the fact that the initial value of fractional-order systems is determined by all past states, it is not simple to take into account the initial condition in a coherent way. In spite of such difficulty, the initialization problems have been studied using Caputo and Riemann-Liouville definitions in Lorenzo and Hartley $(2008,2011)$. The fractional derivatives definitions differ in the initial conditions considered in each case. In the following, well-known two definitions are given with initialization functions.

The Riemann-Liouville derivative with fractional-order $\alpha$ of continuous function $f(t)$ with respect to $t$ and the terminal value 0 is defined as (Lorenzo and Hartley, 2008)

$$
\begin{aligned}
{ }_{0} D_{t}^{\alpha} f(t) & =\frac{1}{\Gamma(n-\alpha)} \frac{\mathrm{d}^{n}}{\mathrm{~d} t^{n}} \int_{a}^{t} \frac{f(\tau)}{(t-\tau)^{\alpha-n+1}} \mathrm{~d} \tau-{ }^{R L} \psi(f, \alpha, a, 0, t), \\
{ }^{R L} \psi(f, \alpha, a, 0, t) & =\frac{1}{\Gamma(n-\alpha)} \frac{\mathrm{d}^{n}}{\mathrm{~d} t^{n}} \int_{a}^{0} \frac{f(\tau)}{(t-\tau)^{\alpha-n+1}} \mathrm{~d} \tau .
\end{aligned}
$$

The Caputo derivative is defined as follows (Lorenzo and Hartley, 2011):

$$
\begin{aligned}
{ }_{0} D_{t}^{\alpha} f(t) & =\frac{1}{\Gamma(n-\alpha)} \int_{a}^{t} \frac{\left(\mathrm{d}^{n} f(\tau) / \mathrm{d} t^{n}\right)}{(t-\tau)^{\alpha-n+1}} \mathrm{~d} \tau-{ }^{C} \psi(f, \alpha, a, 0, t), \\
{ }^{C} \psi(f, \alpha, a, 0, t) & =\frac{1}{\Gamma(n-\alpha)} \int_{a}^{0} \frac{\left(\mathrm{d}^{n} f(\tau) / \mathrm{d} t^{n}\right)}{(t-\tau)^{\alpha-n+1}} \mathrm{~d} \tau,
\end{aligned}
$$

where $n \in \mathbb{N}, \alpha \in \mathbb{R}^{+}, a \in \mathbb{R}^{-}$is the origin of time, $\Gamma$ (.) is the Gamma function and $n-1<\alpha<n$. ${ }^{C} \psi(f, \alpha, a, 0, t)$ and ${ }^{R L} \psi(f, \alpha, a, 0, t)$ are initialization functions for the Caputo definition and the Riemann-Liouville definition, respectively.

In Lee et al. (2014), it has been shown that the definitions of Caputo and Riemann-Liouville derivatives, and their Laplace transforms are equivalent, respectively, when the initial value history is a constant for $a<t_{I}<0$. In the rest of the paper, we use an operator $D^{\alpha} f(t)$ and an initialization function $\psi(f, \alpha, a, 0, t)$ to represent both the Caputo and Riemann-Liouville derivatives with a constant initial value history (i.e., zero initial value history). 
In Trigeassou et al. (2012), Sabatier et al. (2010a) and Hartley et al. (2013), it has been shown that the initialization function approach and the infinite-state approach are strictly equivalent, so we adopted the definitions of fractional derivatives with initialization functions in our paper.

\subsection{Numerical solution of fractional differential equations}

In Diethelm et al. (2005), Diethelm et al. propose a review of the numerical solution techniques for systems of fractional differential equations. The predictor-corrector method proposed by Diethelm et al. (2002) is used in this paper. Such a method is a generalization of the Adams-Bashforth-Moulton predictor-corrector technique suitable for fractional differential equations, the numerical algorithm is based on the Caputo definition for the fractional derivative. The technique can be applied to both linear and non-linear fractional differential systems with either homogenous or non-homogenous initial conditions. In summary, the predictor step is calculated through the following equation (Diethelm et al., 2002):

$$
y_{N}^{p}\left(t_{n+1}\right)=\sum_{k=0}^{[\alpha]-1} \frac{t_{n+1}^{k}}{k !} y_{0}^{(k)}+\frac{1}{\Gamma(\alpha)} \sum_{j=0}^{n} b_{j, n+1} f\left(t_{j}, y_{h}\left(t_{j}\right)\right),
$$

where

$$
b_{j, n+1}=\frac{h^{\alpha}}{\alpha}\left((n+1-j)^{\alpha}-(n-j)^{\alpha}\right),
$$

and the corrector step is calculated by the following equation:

$$
y_{h}\left(t_{n+1}\right)=\sum_{k=0}^{[\alpha]-1} \frac{t_{n+1}^{k}}{k !} y_{0}^{(k)}+\frac{h^{\alpha}}{\Gamma(\alpha+2)} f\left(t_{n+1}, y_{h}^{p}\left(t_{n+1}\right)\right)+\frac{h^{\alpha}}{\Gamma(\alpha+2)} \sum_{j=0}^{n} a_{j, n+1} f\left(t_{j}, y_{h}\left(t_{j}\right)\right),
$$

where

$$
a_{j, n+1}= \begin{cases}n^{\alpha+1}-(n-\alpha)(n+1)^{\alpha}, & \text { if } j=0 \\ (N-j+2)^{\alpha+1}+(n-j)^{\alpha+1}-2(n-j+1)^{\alpha+1}, & \text { if } 1<j<n \\ 1, & \text { if } j=n+1 .\end{cases}
$$

Then, the fractional Adams-Bashforth-Moulton algorithm technique is described by equations (2.2) and (2.4) with the weights defined by equations (2.3) and (2.5).

\subsection{Definition of the two-parameter Mittag-Leffler function}

The two-parameter Mittag-Leffler function plays a very important role in the fractional calculus and is defined in Podlubny (1999) and Erdélyi and Bateman (1955) as follows:

$$
E_{\alpha, \beta}(z)=\sum_{k=0}^{\infty} \frac{z^{k}}{\Gamma(\alpha k+\beta)}, \quad \alpha>0, \quad \beta>0,
$$

where

$$
E_{\alpha, 1}(z)=\sum_{k=0}^{\infty} \frac{z^{k}}{\Gamma(\alpha k+1)} \equiv E_{\alpha}(z)
$$

is the one-parameter Mittag-Leffler function. 
The Laplace transform of the two-parameter Mittag-Leffler function is

$$
\int_{0}^{\infty} e^{-s t} t^{\alpha k+\beta-1} E_{\alpha, \beta}^{(k)}\left(a t^{\alpha}\right) \mathrm{d} t=\frac{s^{\alpha-\beta} k !}{\left(s^{\alpha}-a\right)^{k+1}},
$$

where $E_{\alpha, \beta}^{(k)}=\left(\mathrm{d}(k) / \mathrm{d} t^{k}\right) E_{\alpha, \beta}$.

The use of the Laplace transform of the Mittag-Leffler function (2.6) is a convenient way for obtaining various useful relationships for the Mittag-Leffler function.

Lemma 2.1 (Podlubny, 1999; Wen et al., 2008) If $A \in \mathbb{C}^{n \times n}$ and $\alpha<2, \beta$ is an arbitrary real number, $\gamma$ is such that $\alpha \pi / 2<\gamma<\min [\pi, \pi \alpha]$ and $\theta>0$ is a real constant, then

$$
\left\|E_{\alpha, \beta}(A)\right\| \leqslant \frac{\theta}{1+\|A\|}, \quad \gamma \leqslant\left|\arg \left(\lambda_{i}(A)\right)\right| \leqslant \pi, \quad i=1, \ldots, n,
$$

where $\theta=\max \left(C,\|P\|\left\|P^{-1}\right\| C\right), \lambda_{i}(A)$ denotes the $i$ th eigenvalue of matrix $A, P$ is a non-singular coordinate transformation giving the Jordan form of $A, C /(1+\|A\|) \geqslant \max _{1 \leqslant i \leqslant n}\left|C_{0_{i}} /\left(1+\lambda_{i}\right)\right|$, where $C$ and $C_{0_{i}}$ are given positive constants.

\section{Stability results for fractional-order dynamic systems}

It has been shown that a fractional-order system of order $0<\alpha<2$ is stable if the following condition is satisfied (see Matignon, 1996, 1998) for $0<\alpha \leqslant 1$ (see Sabatier et al., 2008) for $1<\alpha<2$

$$
|\arg (\operatorname{spec}(A))|>\alpha \frac{\pi}{2},
$$

where $\operatorname{spec}(A)$ represents the eigenvalues of matrix $A$.

This paper focuses on the stabilization problems for the case $0<\alpha<1$ and the condition given in Lemma 2.1 is satisfied by (3.1).

The necessary and sufficient LMIs conditions to satisfy condition (3.1) when the fractional-order $\alpha$ belonging to $0<\alpha<1$ are given in the following lemma.

Lemma 3.1 (Lu and Chen, 2010) Let $A \in \mathbb{R}^{n \times n}$ be a real matrix and $0<\alpha<1$. A fractional-order system is asymptotically stable (i.e. $|\arg (\operatorname{spec}(A))|>\alpha(\pi / 2))$ if and only if there exist two real symmetric matrices $P_{k 1} \in \mathbb{R}^{n \times n}, k=1,2$, and two skew-symmetric matrices $P_{k 2} \in \mathbb{R}^{n \times n}, k=1,2$, such that

$$
\begin{aligned}
& \sum_{i=1}^{2} \sum_{j=1}^{2} \operatorname{Sym}\left\{\Gamma_{i j} \otimes\left(A P_{i j}\right)\right\}<0, \\
& {\left[\begin{array}{cc}
P_{11} & P_{12} \\
-P_{12} & P_{11}
\end{array}\right]>0, \quad\left[\begin{array}{cc}
P_{21} & P_{22} \\
-P_{22} & P_{21}
\end{array}\right]>0,}
\end{aligned}
$$




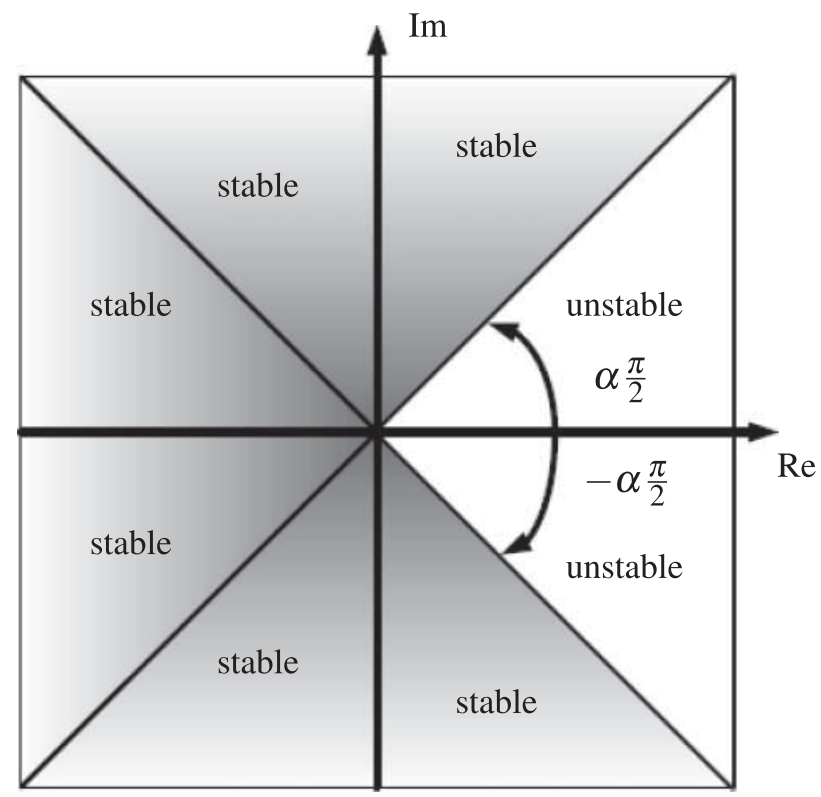

FIG. 1. Stability region of linear fractional-order systems with $0<\alpha<1$.

where

$$
\begin{array}{cc}
\Gamma_{11}=\left[\begin{array}{cc}
\sin \left(\alpha \frac{\pi}{2}\right) & -\cos \left(\alpha \frac{\pi}{2}\right) \\
\cos \left(\alpha \frac{\pi}{2}\right) & \sin \left(\alpha \frac{\pi}{2}\right)
\end{array}\right], \quad \Gamma_{12}=\left[\begin{array}{cc}
\cos \left(\alpha \frac{\pi}{2}\right) & \sin \left(\alpha \frac{\pi}{2}\right) \\
-\sin \left(\alpha \frac{\pi}{2}\right) & \cos \left(\alpha \frac{\pi}{2}\right)
\end{array}\right], \\
\Gamma_{21}=\left[\begin{array}{cc}
\sin \left(\alpha \frac{\pi}{2}\right) & \cos \left(\alpha \frac{\pi}{2}\right) \\
-\cos \left(\alpha \frac{\pi}{2}\right) & \sin \left(\alpha \frac{\pi}{2}\right)
\end{array}\right], \quad \Gamma_{22}=\left[\begin{array}{cc}
-\cos \left(\alpha \frac{\pi}{2}\right) & \sin \left(\alpha \frac{\pi}{2}\right) \\
-\sin \left(\alpha \frac{\pi}{2}\right) & -\cos \left(\alpha \frac{\pi}{2}\right)
\end{array}\right] .
\end{array}
$$

Note that the conditions given in Lemma 3.1 are equivalent to those given in Sabatier et al. (2010b) and Farges et al. (2010). Figure 1 shows the stable and unstable regions of the complex plane for $0<\alpha<1$.

To prove the results in Sections 5 and 6, we need the following lemma.

Lemma 3.2 (Khargonakar et al., 1990) Let $X$ and $Y$ be real vectors of the same dimension. Then, for any scalar $\delta>0$, the following inequality holds

$$
X^{\mathrm{T}} Y+Y^{\mathrm{T}} X \leqslant \delta X^{\mathrm{T}} X+\delta^{-1} Y^{\mathrm{T}} Y .
$$




\section{Uncertain non-linear fractional-order systems}

In this paper, we generalize the integer-order mathematical model to the following uncertain fractionalorder mathematical model where the system was initialized in the past with zero initial value history

$$
\begin{gathered}
\left(A_{n}+\Delta_{n}\right) D_{t}^{n \alpha} q+\cdots+\left(A_{1}+\Delta_{1}\right) D_{t}^{\alpha} q+\left(A_{0}+\Delta_{0}\right) q \\
=\bar{B} u+\bar{f}\left(q, D_{t}^{\alpha} q, \ldots, D_{t}^{(n-1) \alpha} q\right)+\psi(q, \alpha, a, 0, t),
\end{gathered}
$$

where $n$ is an integer, $0<\alpha \leqslant 1, q$ is a vector $\in \mathbb{R}^{n}, A_{i} \in \mathbb{R}^{n \times n}$ and $\bar{B} \in \mathbb{R}^{n \times m}$ are known matrices that represent the values of the system at nominal working point, the vector $u \in \mathbb{R}^{m}$ is a known driving source and $\bar{f}(.) \in \mathbb{R}^{n}$ is a known vector field. $q^{(i)}=\left(q_{1}^{(i)} q_{2}^{(i)} \ldots q_{n}^{(i)}\right)^{\mathrm{T}}$ represents the $i$ th order differentiation of vector $q . \Delta_{i} \in \mathbb{R}^{n \times n}(i=0 \ldots n)$ is the unknown constant matrix describing the uncertain parameters. Note that model (4.1) with the non-linear functions $\bar{f}()=$.0 has been used in Xu and Darouach (1998) with $\alpha=1$ and in Xing and Lu (2009) with $1 \leqslant \alpha<2$.

Suppose that the matrix $\left(A_{n}+\Delta_{n}\right)$ is non-singular (see Xu and Darouach, 1998; Xing and Lu, 2009) (it is true for most of the physical systems), then (4.1) with zero initial value history can be written in the following form:

$$
D^{\alpha} x(t)=\left(I+\Delta_{m}\right)\left[\left(A+\Delta_{a}\right) x(t)+f(x(t)) u(t)+B u(t)+\psi(x, \alpha, a, 0, t)\right], \quad 0<\alpha<1,
$$

where

$$
\begin{aligned}
& A=\left[\begin{array}{cccc}
0 & I_{n} & \ldots & 0 \\
\vdots & \vdots & \ddots & \vdots \\
0 & 0 & \ldots & I_{n} \\
-A_{n}^{-1} A_{0} & -A_{n}^{-1} A_{1} & \ldots & -A_{n}^{-1} A_{n-1}
\end{array}\right], \quad \Delta_{m}=\left[\begin{array}{cc}
0_{(n-1) n \times(n-1) n} & 0 \\
0 & -\left(A_{n}+\Delta_{n}\right)^{-1} \Delta_{n}
\end{array}\right], \\
& B=\left[\begin{array}{c}
0_{(n-1) n \times m} \\
A_{n}^{-1} \bar{B}
\end{array}\right], \quad x=\left[\begin{array}{c}
q \\
D_{t}^{\alpha} q \\
\vdots \\
D_{t}^{(n-1) \alpha} q
\end{array}\right], \quad \Delta_{a}=\left[\begin{array}{ccc}
0_{(n-1) n \times n} & \ldots & 0_{(n-1) n \times n} \\
-A_{n}^{-1} \Delta_{0} & \ldots & -A_{n}^{-1} \Delta_{n-1}
\end{array}\right], \quad f(x)=\left[\begin{array}{c}
0_{(n-1) n \times m} \\
A_{n}^{-1} \bar{f}(x)
\end{array}\right],
\end{aligned}
$$

where $x(t) \in \mathbb{R}^{n^{2}}$ is the pseudo-state vector and $\psi(x, \alpha, a, 0, t)$ is the initialization function.

In the sequel of the paper, we use the following assumption.

Assumption 4.1 The non-linear system (4.2) satisfies the following conditions:

1. The system was initialized in the past, i.e. $x(t)=x_{0}^{\psi}$ for $\left.\left.t \in\right]-\infty, 0\right]$ where $x_{0}^{\psi}$ is a constant vector.

2. The function $f(x(t))$ is bounded and measurable with $f(0)=0$.

3. There exist a positive constant $\mu$ such that

$$
\|f(x(t))\| \leqslant \mu\|x(t)\| .
$$

4. The pair $(A, B)$ is stabilizable. 
REMARK 4.1 The representation (4.2) is not strictly a state space representation, since whatever definition of fractional derivative operator is used, the initial state vector $x(t)$ at the initial time $t_{0}$ is not sufficient to determine the future and past behaviours of the systems. That is the reason why we denote the representation (4.2) as the pseudo-state space representation. We refer the reader to articles Sabatier et al. (2010a, 2012) and Trigeassou et al. (2012).

REMARK 4.2 From the viewpoint of robustness analysis, it is only needed to consider the following two kinds of uncertain systems:

$$
D^{\alpha} x(t)=\left(A+\Delta_{A}\right) x(t)+f(x(t)) u(t)+B u(t)+\psi(x, \alpha, a, 0, t), \quad 0<\alpha<1
$$

and

$$
D^{\alpha} x(t)=\left(I+\Delta_{m}\right)\left[\left(A+\Delta_{a}\right) x(t)+f(x(t)) u(t)+B u(t)+\psi(x, \alpha, a, 0, t)\right], \quad 0<\alpha<1 .
$$

The uncertain fractional-order non-linear systems described by (4.4) is a special case of (4.5) (when $\Delta_{m}=0$ ).

Assumption 4.2 (Xing and Lu, 2009) Consider the fractional-order non-linear systems (4.2) with non-linear uncertain parameters $\Delta_{m}$ and $\Delta_{a}$, where $\Delta_{m s}=-\left(A_{n}+\Delta_{n}\right)^{-1} \Delta_{n}$ and $\Delta_{a}^{i}=-A^{-1} \Delta_{i-1}$ (for $i=1, \ldots, n$ ).

Suppose that

$$
\Delta_{m s}<d_{m}, \quad \Delta_{a}^{i}<d_{a}^{i},
$$

where $d_{m}, d_{a}^{i} \in \mathbb{R}^{n \times n}$ (for $i=1, \ldots, n$ ), then $\Delta_{m}$ and $\Delta_{a}$ satisfy

$$
\begin{aligned}
\Delta_{m} & =\left[\begin{array}{ccc}
0 & \cdots & 0 \\
\vdots & \ddots & \vdots \\
0 & \cdots & \Delta_{m s}
\end{array}\right]<\left[\begin{array}{ccc}
0 & \cdots & 0 \\
\vdots & \ddots & \vdots \\
0 & \cdots & d_{m}
\end{array}\right]=\bar{D}_{m}, \\
\Delta_{a} & =\left[\begin{array}{ccc}
0 & \cdots & 0 \\
\vdots & \ddots & \vdots \\
\Delta_{a}^{1} & \cdots & \Delta_{a}^{n}
\end{array}\right]<\left[\begin{array}{ccc}
0 & \cdots & 0 \\
\vdots & \ddots & \vdots \\
d_{a}^{1} & \cdots & d_{a}^{n}
\end{array}\right]=\bar{D}_{a} .
\end{aligned}
$$

First, to investigate the robust stabilization of the non-linear fractional systems with $0<\alpha<1$, Lemma 3.1 is used to derive a sufficient robust asymptotical stabilization of the linear fractional-order systems in terms of LMIs formulation. Thus, obtained result will be extended to ensure the robust stabilization of the non-linear fractional systems using the generalization Gronwall-Bellman lemma (see N'Doye et al., 2009a,b, 2011).

\section{Robust stabilization of linear fractional-order systems}

Consider the linear fractional-order system with non-linear uncertain parameters $\Delta_{m}$ and $\Delta_{a}$ described by the pseudo-state space representation equation of the following form $((4.2)$ with $f()=0$.

$$
D^{\alpha} x(t)=\left(I+\Delta_{m}\right)\left[\left(A+\Delta_{a}\right) x(t)+B u(t)+\psi(x, \alpha, a, 0, t)\right], \quad 0<\alpha<1,
$$


where $x(t) \in \mathbb{R}^{n}$ is the state vector, $u(t) \in \mathbb{R}^{m}$ is the control input vector and $\psi(x, \alpha, a, 0, t) \in \mathbb{R}^{n}$ is the initialization function. $A$ and $B$ are known constant matrices, $\Delta_{m}$ and $\Delta_{a}$ are defined in Assumption 4.2. The system was initialized in the past, i.e. $x(t)=x_{0}^{\psi}$ for $\left.\left.t \in\right] a, 0\right]$ where $x_{0}^{\psi}$ is a constant vector.

The asymptotical stabilization of system (5.1) is given in the following theorem.

Theorem 5.1 Under Assumptions 4.1 and 4.2, the linear fractional-order system (5.1) where $0<\alpha<1$ with non-linear uncertain parameters $\Delta_{m}$ and $\Delta_{a}$ satisfying (4.7) and $\psi(x, \alpha, a, 0, t)=0$ controlled by the following feedback:

$$
u(t)=L x(t)
$$

is asymptotically stable if there exists a matrix $Y \in \mathbb{R}^{m \times n}$ and a symmetric positive-definite matrix $N \in \mathbb{R}^{n \times n}$ and four real scalars $\delta_{i}>0$ and $\ell_{i}>0,(i=1,2)$ such that

$$
\Xi=\left[\begin{array}{ll}
\Xi_{11} & \Xi_{12} \\
\Xi_{12}^{\mathrm{T}} & \Xi_{22}
\end{array}\right]<0,
$$

where

$$
\begin{aligned}
& \Xi_{11}=\sum_{i=1}^{2} \operatorname{Sym}\left\{\Gamma_{i 1} \otimes(A N+B Y)\right\}+\sum_{i=1}^{2} \delta_{i}\left(I_{2} \otimes \widehat{D}_{m}\right)+\sum_{i=1}^{2} \ell_{i}\left(I_{2} \otimes \widehat{D}_{m a}\right)
\end{aligned}
$$

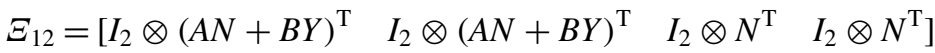

$$
\begin{aligned}
& \Xi_{22}=-\operatorname{diag}\left(\delta_{1}, \delta_{2}, \ell_{1}, \ell_{2}\right) \otimes I_{2 n}
\end{aligned}
$$

and $\Gamma_{i 1}(i=1,2)$ satisfy $(3.4)$.

Moreover, a stabilizing state-feedback gain matrix is given by $L=Y N^{-1}$.

Proof. The linear fractional-order systems (5.1) with the linear feedback $u(t)=L x(t)$ can be written as

$$
D^{\alpha} x(t)=\widetilde{A} x(t), \quad 0<\alpha<1,
$$

where $\widetilde{A}=\left(I+\Delta_{m}\right)\left[\left(A+\Delta_{a}\right)+B L\right]=(A+B L)+\Delta_{m}(A+B L)+\Delta_{a}+\Delta_{a} \Delta_{m}$.

From relation (4.6) (see Xing and $\mathrm{Lu}, 2009$ ), it is easy to see that

$$
\Delta_{m} \Delta_{m}^{\mathrm{T}} \leqslant \widehat{D}_{m} \quad \text { and } \quad \Delta_{m a} \Delta_{m a}^{\mathrm{T}} \leqslant \widehat{D}_{m a}
$$

where $\Delta_{m a}=\Delta_{a}+\Delta_{m} \Delta_{a}$ and $\widehat{D}_{m}, \widehat{D}_{m a} \in \mathbb{R}^{n \times n}$.

The linear fractional-order systems (5.1) are asymptotically stabilizable if the fractional-order system (5.4) are asymptotically stable. It follows from Lemma 3.1 that this is equivalent to that there exist two real symmetric matrices $P_{k 1} \in \mathbb{R}^{n \times n}, k=1,2$, and two skew-symmetric matrices $P_{k 2} \in \mathbb{R}^{n \times n}$, $k=1,2$, such that

$$
\begin{aligned}
\sum_{i=1}^{2} \sum_{j=1}^{2} \operatorname{Sym}\left\{\Gamma_{i j} \otimes\left(\widetilde{A} P_{i j}\right)\right\}= & \sum_{i=1}^{2} \sum_{j=1}^{2} \operatorname{Sym}\left\{\Gamma_{i j} \otimes\left(\bar{A} P_{i j}\right)\right\} \\
& +\sum_{i=1}^{2} \sum_{j=1}^{2} \operatorname{Sym}\left\{\Gamma_{i j} \otimes\left(\Delta_{m} \bar{A} P_{i j}+\Delta_{m a} P_{i j}\right)\right\}<0,
\end{aligned}
$$

where $\bar{A}=A+B L$ and $\Gamma_{i j}(i, j=1,2)$ satisfy (3.4). 
Now, taking $P_{11}=P_{21}=N$ and $P_{12}=P_{22}=0$ in relation (5.6), we obtain the following relation:

$$
\begin{aligned}
\sum_{i=1}^{2} \sum_{j=1}^{2} \operatorname{Sym}\left\{\Gamma_{i j} \otimes\left(\widetilde{A} P_{i j}\right)\right\}= & \sum_{i=1}^{2} \operatorname{Sym}\left\{\Gamma_{i 1} \otimes(\bar{A} N)\right\} \\
& +\sum_{i=1}^{2} \operatorname{Sym}\left\{\Gamma_{i 1} \otimes\left(\Delta_{m} \bar{A} N+\Delta_{m a} N\right)\right\}<0 .
\end{aligned}
$$

Note that $\Gamma_{i 1} \Gamma_{i 1}^{\mathrm{T}}=I_{2}(i=1,2)$ and it follows from Lemma 3.2 that for any real scalars $\delta_{i}>0$ and $\ell_{i}>0,(i=1,2)$ such that

$$
\begin{aligned}
\sum_{i=1}^{2} & \operatorname{Sym}\left\{\Gamma_{i 1} \otimes\left(\Delta_{m} \bar{A} N+\Delta_{m a} N\right)\right\} \\
= & \sum_{i=1}^{2} \operatorname{Sym}\left\{\Gamma_{i 1} \otimes\left(\Delta_{m} \bar{A} N\right)\right\}+\sum_{i=1}^{2} \operatorname{Sym}\left\{\Gamma_{i 1} \otimes\left(\Delta_{m a} N\right)\right\} \\
= & \sum_{i=1}^{2} \operatorname{Sym}\left\{\left(\Gamma_{i 1} \otimes \Delta_{m}\right)\left(I_{2} \otimes \bar{A} N\right)\right\}+\sum_{i=1}^{2} \operatorname{Sym}\left\{\left(\Gamma_{i 1} \otimes \Delta_{m a}\right)\left(I_{2} \otimes N\right)\right\}, \\
\leqslant & \sum_{i=1}^{2} \delta_{i}\left(\Gamma_{i 1} \otimes \Delta_{m}\right)\left(\Gamma_{i 1} \otimes \Delta_{m}\right)^{\mathrm{T}}+\sum_{i=1}^{2} \delta_{i}^{-1}\left(I_{2} \otimes \bar{A} N\right)^{\mathrm{T}}\left(I_{2} \otimes \bar{A} N\right) \\
& +\sum_{i=1}^{2} \ell_{i}\left(\Gamma_{i 1} \otimes \Delta_{m a}\right)\left(\Gamma_{i 1} \otimes \Delta_{m a}\right)^{\mathrm{T}}+\sum_{i=1}^{2} \ell_{i}^{-1}\left(I_{2} \otimes N\right)^{\mathrm{T}}\left(I_{2} \otimes N\right), \\
\leqslant & \sum_{i=1}^{2} \delta_{i}\left(I_{2} \otimes \Delta_{m} \Delta_{m}^{\mathrm{T}}\right)+\sum_{i=1}^{2} \delta_{i}^{-1}\left(I_{2} \otimes \bar{A} N\right)^{\mathrm{T}}\left(I_{2} \otimes \bar{A} N\right) \\
& +\sum_{i=1}^{2} \ell_{i}\left(I_{2} \otimes \Delta_{m a} \Delta_{m a}^{\mathrm{T}}\right)+\sum_{i=1}^{2} \ell_{i}^{-1}\left(I_{2} \otimes N\right)^{\mathrm{T}}\left(I_{2} \otimes N\right) .
\end{aligned}
$$

From (5.5), the inequality (5.8) can be written

$$
\begin{aligned}
\sum_{i=1}^{2} \operatorname{Sym}\left\{\Gamma_{i 1} \otimes\left(\Delta_{m} \bar{A} N+\Delta_{m a} N\right)\right\} \leqslant \sum_{i=1}^{2} & \delta_{i}\left(I_{2} \otimes \widehat{D}_{m}\right)+\sum_{i=1}^{2} \delta_{i}^{-1}\left(I_{2} \otimes \bar{A} N\right)^{\mathrm{T}}\left(I_{2} \otimes \bar{A} N\right) \\
& +\sum_{i=1}^{2} \ell_{i}\left(I_{2} \otimes \widehat{D}_{m a}\right)+\sum_{i=1}^{2} \ell_{i}^{-1}\left(I_{2} \otimes N\right)^{\mathrm{T}}\left(I_{2} \otimes N\right) .
\end{aligned}
$$


Substituting (5.9) into (5.7), we obtain

$$
\begin{aligned}
\sum_{i=1}^{2} \sum_{j=1}^{2} \operatorname{Sym}\left\{\Gamma_{i j} \otimes\left(\widetilde{A} P_{i j}\right)\right\} \leqslant & \sum_{i=1}^{2} \operatorname{Sym}\left\{\Gamma_{i 1} \otimes(\bar{A} N)\right\}+\sum_{i=1}^{2} \delta_{i}\left(I_{2} \otimes \widehat{D}_{m}\right) \\
& +\sum_{i=1}^{2} \delta_{i}^{-1}\left(I_{2} \otimes \bar{A} N\right)^{\mathrm{T}}\left(I_{2} \otimes \bar{A} N\right)+\sum_{i=1}^{2} \ell_{i}\left(I_{2} \otimes \widehat{D}_{m a}\right) \\
& +\sum_{i=1}^{2} \ell_{i}^{-1}\left(I_{2} \otimes N\right)^{\mathrm{T}}\left(I_{2} \otimes N\right)<0 .
\end{aligned}
$$

Set $Y=L N$ and substitute $\bar{A}=A+B L$ into (5.10), we have

$$
\begin{aligned}
\sum_{i=1}^{2} \sum_{j=1}^{2} \operatorname{Sym}\left\{\Gamma_{i j} \otimes\left(\widetilde{A} P_{i j}\right)\right\} \leqslant & \sum_{i=1}^{2} \operatorname{Sym}\left\{\Gamma_{i 1} \otimes(A N+B Y)\right\}+\sum_{i=1}^{2} \delta_{i}\left(I_{2} \otimes \widehat{D}_{m}\right)+\sum_{i=1}^{2} \ell_{i}\left(I_{2} \otimes \widehat{D}_{m a}\right) \\
& +\sum_{i=1}^{2} \delta_{i}^{-1}\left(I_{2} \otimes(A N+B Y)\right)^{\mathrm{T}}\left(I_{2} \otimes(A N+B Y)\right) \\
& +\sum_{i=1}^{2} \ell_{i}^{-1}\left(I_{2} \otimes N\right)^{\mathrm{T}}\left(I_{2} \otimes N\right)<0
\end{aligned}
$$

This inequality (5.11) is equivalent to (5.3) by the Schur complement (see Boyd et al., 1994). This completes the proof.

In Section 5, a sufficient condition for the linear fractional-order systems to be robustly asymptotically stable is studied. Now, in Section 6, a sufficient robust stabilization condition for the non-linear fractional-order systems is derived using the generalization of Gronwall-Bellman approach.

\section{Robust stabilization of non-linear fractional-order systems}

Consider the non-linear fractional-order system with non-linear uncertain parameters $\Delta_{m}$ and $\Delta_{a}$ described by the following form:

$$
D^{\alpha} x(t)=\left(I+\Delta_{m}\right)\left[\left(A+\Delta_{a}\right) x(t)+f(x(t)) u(t)+B u(t)+\psi(x, \alpha, a, 0, t)\right], \quad 0<\alpha<1,
$$

where $x(t) \in \mathbb{R}^{n}$ is the state vector and $u(t) \in \mathbb{R}^{m}$ is the control input vector. $A$ and $B$ are known constant matrices, $\Delta_{m}$ and $\Delta_{a}$ are defined in Assumption 4.2.

Under Assumptions 4.1 and 4.2, the robust asymptotical stabilization of system (6.1) is given in the following theorem.

Theorem 6.1 Consider that Assumptions 4.1 and 4.2 hold.

First, assume that the non-linear function $f(x(t))=0$. Then, the non-linear fractional-order system (6.1) with non-linear uncertain parameters $\Delta_{m}$ and $\Delta_{a}$ satisfying (4.7) controlled by the following 
feedback:

$$
u(t)=L x(t)
$$

is robustly asymptotically stable.

Secondly, assume that the non-linear function $f(x(t))$ is not equal to zero. Then, the non-linear fractional-order system (6.1) with non-linear uncertain parameters $\Delta_{m}$ and $\Delta_{a}$ satisfying (4.7) controlled by the feedback (6.2) is robustly asymptotically stable and has a unique solution $x(t)$. In addition, the state $x(t)$ is bounded as follows:

$$
\|x(t)\| \leqslant \frac{\theta\left\|x_{0}\right\| /\left(1+\|\widetilde{A}\| t^{\alpha}\right)}{\left(1-\left(\left(2 \mu \theta^{2}\|L\|\left\|x_{0}\right\|\right) /(\alpha\|\widetilde{A}\|)\right)(1-(1 /(1+\|\widetilde{A}\|(t / 2))))\right)},
$$

where the initial state $\left\|x_{0}\right\|$ satisfy

$$
\left\|x_{0}\right\|<\left(\frac{\alpha\|\widetilde{A}\|}{2 \mu\|L\| \theta^{2}}\right)
$$

Moreover, a stabilizing state-feedback gain matrix is given by $L=Y N^{-1}$.

Proof. Consider that Assumption 4.2 hold. If $f(x(t))=0$, the proof of the robust asymptotical stabilization using the feedback control (6.2) is given by Theorem 5.1.

Now, assume that the initial state $\left\|x_{0}\right\|$ satisfy relation (6.4) and the non-linear function $f(x(t))$ is not equal to zero and satisfy Assumption 4.1.

The non-linear fractional-order system (4.2) with the control feedback $u(t)=L x(t)$ can be written as

$$
D^{\alpha} x(t)=\left(I+\Delta_{m}\right)\left[\left(A+\Delta_{a}\right)+B L\right] x(t)+f(x(t)) L x(t)+\psi(x, \alpha, a, 0, t), \quad 0<\alpha<1,
$$

where $\widetilde{A}=\left(I+\Delta_{m}\right)\left[\left(A+\Delta_{a}\right)+B L\right], \Delta_{m}$ and $\Delta_{a}$ satisfy the relation (4.7).

Using the Laplace transform on the system (6.5), we obtain

$$
X(s)=\left(I_{n} s^{\alpha}-\widetilde{A}\right)^{-1}[\mathcal{L}(\psi(x, \alpha, a, 0, t))+\mathcal{L}(f(x(t)) L x(t))]
$$

Then, taking the Laplace inverse transform for (6.6), obtained with the inverse Laplace transform formula of the Mittag-Leffler function in two parameters, and using the integral convolution yield

$$
x(t)=E_{\alpha, 1}\left(\widetilde{A} t^{\alpha}\right) x_{0}^{\psi}+\int_{0}^{t}(t-\tau)^{\alpha-1} E_{\alpha, \alpha}\left(\widetilde{A}(t-\tau)^{\alpha}\right) f(x(\tau)) L x(\tau) \mathrm{d} \tau
$$

Using the norm operator on the both sides of (6.7) and from condition (4.3) and Lemma 2.1, we obtain

$$
\|x(t)\| \leqslant \frac{\theta\left\|x_{0}\right\|}{1+\left\|\widetilde{A} t^{\alpha}\right\|}+\int_{0}^{t} \frac{\mu \theta\|L\|\|t-\tau\|^{\alpha-1}}{1+\left\|\widetilde{A}(t-\tau)^{\alpha}\right\|}\|x(\tau)\|^{2} \mathrm{~d} \tau
$$


or equivalently

$$
\|x(t)\| \leqslant \frac{\theta\left\|x_{0}\right\|}{1+\|\widetilde{A}\| t^{\alpha}}+\int_{0}^{t} \frac{\mu \theta\|L\|(t-\tau)^{\alpha-1}}{1+\|\widetilde{A}\|(t-\tau)^{\alpha}}\|x(\tau)\|^{2} \mathrm{~d} \tau .
$$

Then, according to the generalization of Gronwall-Bellman Lemma A.1 (see N'Doye et al., 2009a,b, 2011), let

$$
r(t)=\frac{\theta\left\|x_{0}\right\|}{1+\|\widetilde{A}\| t^{\alpha}}, \quad f(\tau)=\frac{\mu \theta\|L\|(t-\tau)^{\alpha-1}}{1+\|\widetilde{A}\|(t-\tau)^{\alpha}}, \quad \ell=2 .
$$

By the generalization of Gronwall-Bellman lemma, if the following inequality is satisfied

$$
1-(\ell-1) \int_{a}^{t}(r(s))^{\ell-1} f(s) \mathrm{d} s>0,
$$

then, the state is bounded as follows:

$$
x(t) \leqslant \frac{r(t)}{\left(1-(\ell-1) \int_{a}^{t}(r(s))^{\ell-1} f(s) \mathrm{d} s\right)^{1 /(\ell-1)}} .
$$

Substituting (6.10) into (6.11) yields

$$
1-\mu\|L\| \int_{0}^{t}\left(\frac{\theta\left\|x_{0}\right\|}{1+\|\widetilde{A}\| \tau^{\alpha}}\right) \frac{\theta(t-\tau)^{\alpha-1}}{1+\|\widetilde{A}\|(t-\tau)^{\alpha}} \mathrm{d} \tau>0 \quad \forall t>0,
$$

which is equivalent to

$$
1-\mu \theta^{2}\|L\|\left\|x_{0}\right\| \Phi(t)>0 \quad \forall t>0
$$

where

$$
\Phi(t)=\int_{0}^{t} \frac{1}{\left(1+\|\widetilde{A}\| \tau^{\alpha}\right)} \frac{(t-\tau)^{\alpha-1}}{\left(1+\|\widetilde{A}\|(t-\tau)^{\alpha}\right)} \mathrm{d} \tau .
$$

To verify the inequality (6.14), equation (6.15) is rewritten to the sum of the two parts

$$
\Phi(t)=\int_{0}^{t / 2} \frac{1}{\left(1+\|\widetilde{A}\| \tau^{\alpha}\right)} \frac{(t-\tau)^{\alpha-1}}{\left(1+\|\widetilde{A}\|(t-\tau)^{\alpha}\right)} \mathrm{d} \tau+\int_{t / 2}^{t} \frac{1}{\left(1+\|\widetilde{A}\| \tau^{\alpha}\right)} \frac{(t-\tau)^{\alpha-1}}{\left(1+\|\widetilde{A}\|(t-\tau)^{\alpha}\right)} \mathrm{d} \tau
$$

which is equivalent to

$$
\Phi(t)=2 \int_{0}^{t / 2} \frac{\tau^{\alpha-1}}{\left(1+\|\widetilde{A}\| \tau^{\alpha}\right)^{2}} \mathrm{~d} \tau
$$

By integrating (6.17), we obtain

$$
\Phi(t)=\frac{2}{\alpha\|\widetilde{A}\|}\left(1-\frac{1}{1+\|\widetilde{A}\|(t / 2)}\right)
$$


Using (6.18), we have $\Phi(t)>0$ if $t>0$ and the expression

$$
1-\mu\|L\| \theta^{2}\left\|x_{0}\right\| \Phi(t),
$$

in relation (6.14) is minimal when $t$ tends to infinity. Then, inequality (6.14) holds if the initial state $x_{0}$ satisfies condition (6.4). From (6.9), the generalization of Gronwall-Bellman lemma given in Lemma A.1 yields the following inequality:

$$
\|x(t)\| \leqslant \frac{\theta\left\|x_{0}\right\| /\left(1+\|\widetilde{A}\| t^{\alpha}\right)}{\left(1-\left(\left(2 \mu \theta^{2}\|L\|\left\|x_{0}\right\|\right) / \alpha\|\widetilde{A}\|\right)(1-(1 /(1+\|\widetilde{A}\|(t / 2))))\right)},
$$

which is equivalent to inequality (6.3). Then, if time $t$ tends to infinity, $\|x(t)\|$ converges which implies the asymptotic stability of the zero solution. This ends the proof.

\section{Numerical example}

In this section, we provide a numerical example to illustrate the applicability of the proposed method. Considering the following non-linear fractional-order system with non-linear uncertain parameters $\Delta_{m}$ and $\Delta_{a}$

$$
D^{\alpha} x(t)=\left(I+\Delta_{m}\right)\left[\left(A+\Delta_{a}\right) x(t)+f(x(t)) u(t)+B u(t)\right], \quad \alpha=0.76,
$$

where

$$
\begin{gathered}
A=\left[\begin{array}{ccc}
0 & 1 & 0 \\
0 & 0 & 1 \\
1 & -4 & 5
\end{array}\right], \quad B=\left[\begin{array}{l}
0 \\
0 \\
1
\end{array}\right], \quad \Delta_{m}=\left[\begin{array}{ccc}
0 & 0 & 0 \\
0 & 0 & 0 \\
0 & 0 & \delta_{m}
\end{array}\right]<D_{m}=\left[\begin{array}{ccc}
0 & 0 & 0 \\
0 & 0 & 0 \\
0 & 0 & 0.0124
\end{array}\right], \\
\Delta_{a}=\left[\begin{array}{ccc}
0 & 0 & 0 \\
0 & 0 & 0 \\
\delta_{0} & \delta_{1} & \delta_{2}
\end{array}\right]<D_{a}=\left[\begin{array}{ccc}
0 & 0 & 0 \\
0 & 0 & 0 \\
0.01183 & 0.01732 & 0.06512
\end{array}\right], \quad f(x)=\left[\begin{array}{c}
0 \\
-x_{1} x_{3} \\
x_{1} x_{2}
\end{array}\right] .
\end{gathered}
$$

First, assume that the non-linear function $f(x(t))=0$. From Theorem 5.1, it follows that the non-linear fractional-order system (7.1) is asymptotically stable.

A feasible solution of (5.3) for the above fractional-order system (7.1) when $f(x(t))=0$ is obtained as follows:

$$
Y=\left[\begin{array}{lll}
-0.8320 & 0.9897 & -1.3368
\end{array}\right], \quad N=\left[\begin{array}{ccc}
0.2626 & -0.1106 & 0.0259 \\
-0.1106 & 0.1173 & -0.0826 \\
0.0259 & -0.0826 & 0.1393
\end{array}\right],
$$

with $\alpha=0.76, \quad \delta_{1}=1.0467, \quad \delta_{2}=0.2085, \quad \ell_{1}=0.9810 \quad$ and $\quad \ell_{2}=1.0542$.

Finally, the asymptotically stabilizing state-feedback gain matrix can be obtained

$$
L=Y N^{-1}=\left[\begin{array}{lll}
-2.4736 & -0.5712 & -9.4738
\end{array}\right] .
$$

The time response of the system (7.1) with non-linear function $f(x(t))=0$ and $\alpha=0.76$ is shown in Fig. 2, which shows that it is asymptotically stable and its states converge to zero.

From Theorem 6.1, assume that the non-linear function $f(x(t))$ is not equal to zero and the initial state satisfy $\left\|x_{0}\right\| \leqslant 0.0741$ with $\theta=1.865$. 


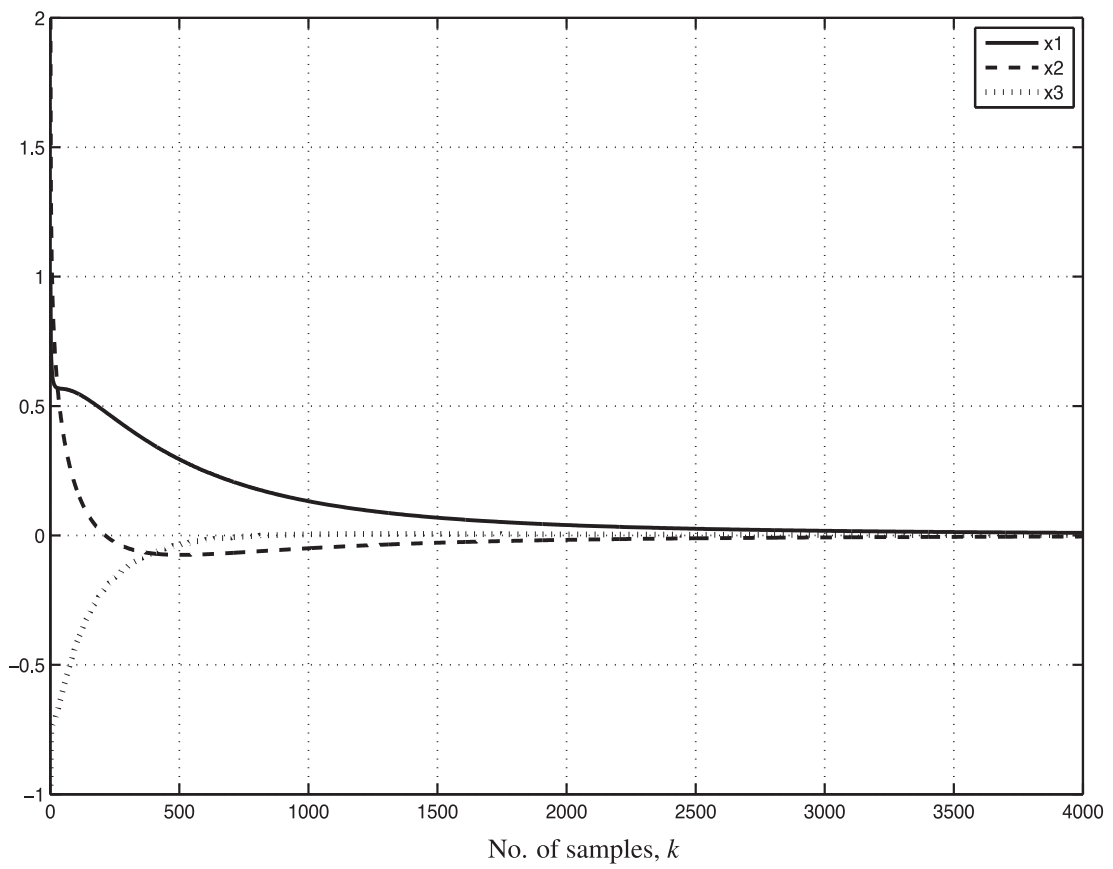

FIG. 2. Time response of the selected system where $f(x(t))=0$ and $\alpha=0.76$.

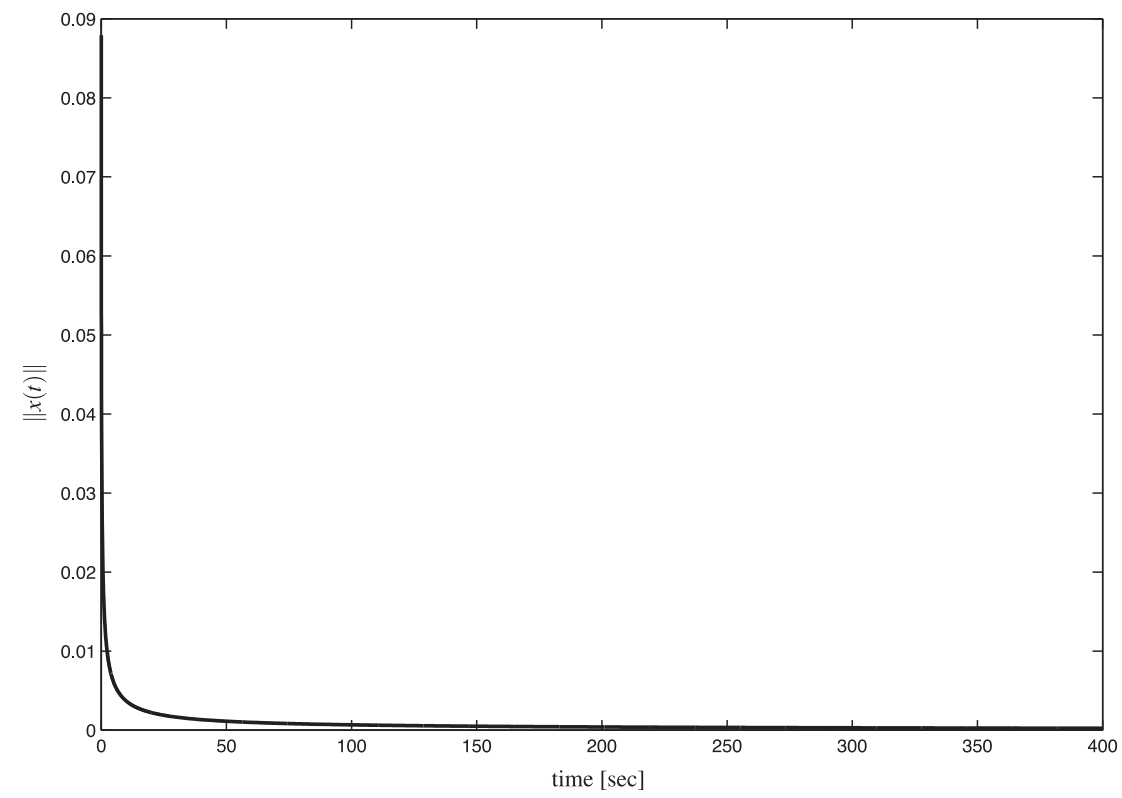

FIG. 3. Time response of the system with $u(t)=L x(t)$. 
Then, the non-linear fractional-order system (7.1) with non-linear uncertain parameters $\Delta_{m}$ and $\Delta_{a}$ controlled by the feedback (6.2) is robustly asymptotically stable and the time response of the system with

$$
\widetilde{A}=\left(I+\Delta_{m}\right)\left[\left(A+\Delta_{a}\right)+B L\right]=\left[\begin{array}{ccc}
0 & 1 & 0 \\
0 & 0 & 1 \\
-1.4799 & -4.6104 & -4.4634
\end{array}\right]
$$

is shown in Fig. 3, which shows that it is asymptotically stable and its states converge to zero.

\section{Conclusion}

In this paper, the stabilization problem of uncertain linear and non-linear fractional-order system with non-linear uncertain parameters has been studied using LMIs in the linear case and using LMIs and the generalization of Gronwall-Bellman lemma in the non-linear case. Sufficient conditions for the robust asymptotical stabilization of such linear and non-linear fractional-order system with non-linear uncertain parameters have been obtained. A numerical example has been given to show the effectiveness of the proposed results.

\section{REFERENCES}

Aghababa, M. P. (2012) Robust stabilization and synchronization of a class of fractional-order chaotic systems via a novel fractional sliding mode controller. Commun. Nonlinear Sci. Numer. Simul., 17, 2670-2681.

Ahn, H., Chen, Y. \& Podlubny, I. (2007) Robust stability test of a class of linear time-invariant interval fractionalorder system using Lyapunov inequality. Appl. Math. Comput., 187, 27-34.

Boyd, S., Féron, E. \& Balakrishnan, V. (1994) Linear Matrix Inequality in Systems and Control Theory. Philadelphia: SIAM.

Chen, Y., Ahn, H. \& Podlubny, I. (2006) Robust stability check of fractional order linear time invariant systems with interval uncertainties. Signal Process., 86, 2611-2618.

Chen, B. \& Wong, C. (1987) Robust linear controller design: time domain approach. IEEE Trans. Autom. Control, 32, 161-164.

DAS, S. (2008) Functional Fractional Calculus for System Identification and Controls. Heidelberg: Springer.

Desoer, C. \& Vidyasagar, M. (1975) Feedback Systems Input-Output Properties. New York: Electrical Sciences. Academic Press.

Diethelm, K., Ford, J. N. \& Freed, D. A. (2002) A predictor-corrector approach for the numerical solution of fractional differential equations. Nonlinear Dyn., 29, 3-22.

Diethelm, K., Ford, J. N., Freed, D. A. \& Luchko, Y. (2005) Algorithms for the fractional calculus: a selection of numerical methods. Comput. Methods Appl. Mech. Eng., 194, 743-773.

Erdélyi, A. \& Bateman, H. (1955) Higher Transcendental Functions, vol. 3. Hightstown, NJ: McGraw-Hill, pp. 43-57.

Farges, C., Moze, M. \& Sabatier, J. (2010) Pseudo-state feedback stabilization of commensurate fractional order systems. Automatica, 46, 1730-1734.

Hartley, T. T., Lorenzo, C. F., Trigeassou, J. C. \& Maamri, N. (2013) Equivalence of history-function based and infinite-dimensional-state initializations for fractional-order operators. ASME J. Comput. Nonlinear Dyn., 8, 041014.

Jetto, L. \& Orsini, V. (2007) Relaxed sufficient conditions for the stability of continuous and discrete-time linear time-varying systems. Proceedings of IEEE Conference on Decision \& Control, New Orleans, USA.

Khargonakar, P., Petersen, I. \& Zhou, K. (1990) Robust stabilization of uncertain linear systems: quadratic stability and $\mathcal{H}_{\infty}$ control theory. IEEE Trans. Autom. Control, 35, 356-361. 
Lee, S. C., Li, Y., Chen, Y. \& Ahn, H. S. (2014) $\mathcal{H}_{\infty}$ and sliding mode observers for linear timeinvariant fractional-order dynamic systems with initial memory effect. J. Dyn. Syst. Meas. Control, 136, 051022 .

Lorenzo, F. C. \& HARTley, T. T. (2008) Initialization of fractional-order operators and fractional differential equations. ASME J. Comput. Nonlinear Dyn., 3, 021101.

Lorenzo, F. C. \& Hartley, T. T. (2011) Time-varying initialization and Laplace transform of the Caputo derivative: with order between zero and one. IDET/CIE FDTA' 2011 Conference, vol. 3, Washington, USA, pp. 163-168.

LU, J. \& CHEN, Y. (2010) Robust stability and stabilization of fractional-order interval systems with the fractionalorder $\alpha$ : the $0<\alpha<1$ case. IEEE Trans. Autom. Control, 55, 152-158.

Matignon, D. (1996) Stability results for fractional differential equations with applications to control processing. Proceedings of IEEE-IMACS Conference on Systems, Man, and Cybernetics, Lille, France.

Matignon, D. (1998) Generalized fractional differential and difference equations: stability properties and modelling issues. Proceedings of Mathematical Theory of Networks and Systems Symposium, Padova, Italy.

N'Doye, I., ZASADZINSKI, M., DAROUACH, M. \& RADHY, N. (2009a) Observer-based control for fractional-order continuous-time systems. Proceedings of IEEE Conference on Decision \& Control, Shanghai, P.R. China.

N'Doye, I., Zasadzinski, M., Darouach, M. \& Radhy, N. (2009b) Stabilization of a class of nonlinear affine fractional-order systems. Proceedings of International IFAC-IEEE Conference on Methods and Models in Automation and Robotics, Miedzyzdroje, Poland.

N'Doye, I., ZASAdZinski, M., DARouACH, M. \& RAdHy, N. (2010) Robust stabilization of linear and nonlinear fractional-order systems with nonlinear uncertain parameters. Proceedings of IEEE Conference on Decision \& Control, Atlanta, USA.

N'Doye, I., Zasadzinski, M., Darouach, M., Radhy, N. \& Bouaziz, A. (2011) Exponential stabilization of a class of nonlinear systems: a generalized Bellman-Gronwall lemma approach. Nonlinear Anal.: Theor. Methods Appl., 74, 7333-7341.

Pachpatte, B. (1973) A note on Gronwall-Bellman inequality. J. Math. Anal. Appl., 44, 758-762.

Petrás̆, I., Chen, Y. \& Vinagre, B. (2004a) Robust stability test for interval fractional-order linear systems. Unsolved Problems in the Mathematics of Systems and Control (V. Blondel \& A. Megretski eds), vol. 38. Princeton: Princeton University Press, pp. 208-210.

Petrášs, I., Chen, Y., Vinagre, B. \& Podlubny, I. (2004b) Stability of linear time invariant systems with interval fractional orders and interval coefficients. International Conference on Computation Cybernetics, Vienna Technical University, Vienna, Austria.

Podlubny, I. (1999) Fractional Differential Equations. New York: Academic Press.

Sabatier, J., Farges, C., Merveillaut, M. \& Feneteau, L. (2012) On observability and pseudo state estimation of fractional order systems. Eur. J. Control, 18, 260-271.

Sabatier, J., Farges, C. \& Trigeassou, J. C. (2013) A stability test for non-commensurate fractional-order systems. Syst. \& Control Lett., 62, 739-746.

Sabatier, J., Merveillaut, M., Malti, R. \& Oustaloup, A. (2010a) How to impose physically coherent initial conditions to a fractional system? Commun. Nonlinear Sci. Numer. Simul., 15, 1318-1326.

Sabatier, J., Moze, M. \& Farges, C. (2008) On stability of fractional order systems. Proceedings of IFAC Workshop on Fractional Differentiation and its Application, Ankara, Turkey.

Sabatier, J., Moze, M. \& Farges, C. (2010b) LMI conditions for fractional order systems. Comput. Math. Appl., 59, 1594-1609.

Shimizu, K. (2000) Nonlinear state observers by gradient descent method. Proceedings of IEEE Conference on Control \& Applications, Anchorage, USA.

Trigeassou, J. C. \& MAAmri, N. (2010) The initial conditions of Riemann-Liouville and Caputo fractional derivatives: an integrator interpretation. Proceedings of IFAC Workshop on Fractional Differentiation and its Application, FDA 10-103. Badajoz, Spain.

Trigeassou, J. C. \& MAAmri, N. (2011) Initial conditions and initialization of linear fractional differential equations. Signal Process., 91, 427-436. 
Trigeassou, J. C., MaAmri, N. \& Oustaloup, A. (2011) Automatic initialization of the Caputo fractional derivative. Proceedings of IEEE Conference on Decision \& Control, Orlando, USA.

Trigeassou, J. C., MaAmRI, N. \& Oustaloup, A. (2013) The infinite state approach: Origin and necessity. Comput. Math. Appl., 66, 892-907.

Trigeassou, J. C., MaAmri, N., Sabatier, J. \& Oustaloup, A. (2012) State variables and transients of fractional order differential systems. Comput. Math. Appl., 64, 3117-3140.

WEN, X., Wu, Z. \& Lu, J. (2008) Stability analysis of a class of nonlinear fractional-order systems. IEEE Trans. Circuits Syst. II: Express Briefs, 55, 1178-1182.

XING, S. \& LU, J. (2009) Robust stability and stabilization of fractional-order linear systems with nonlinear uncertain parameters: an LMI approach. Chaos Solitons Fractals, 42, 1163-1169.

Xu, S. \& Darouach, M. (1998) On the robustness of linear systems with nonlinear uncertain parameters. Automatica, 34, 1005-1008.

$\dot{Z}_{\mathrm{AK}}$, S. (1990) On the stabilization and observation of nonlinear / uncertain dynamic systems. IEEE Trans. Autom. Control, 35, 604-607.

Zevin, A. \& PinsKy, M. (2003) Exponential stability and solution bounds for systems with bounded nonlinearities. IEEE Trans. Autom. Control, 48, 1799-1804.

\section{Appendix}

\section{A.1 Generalization of Gronwall-Bellman lemma}

Lemma A.1 (N'Doye et al., 2009a,b, 2011) Let

(i) $a, b \in \mathbb{R}, 0 \leqslant a<b, r(t)>0$ a positive, monotonic, decreasing function, an integer $\ell>1$,

(ii) $f:[a, b] \mapsto \mathbb{R}^{+}$an integrable function such that, $\forall \alpha, \beta \in[a, b],(0 \leqslant \alpha<\beta)$, we have

$$
\int_{\alpha}^{\beta} f(s) \mathrm{d} s>0,
$$

(iii) $x:[a, b] \mapsto \mathbb{R}^{+}$an essential bounded function such that

$$
x(t) \leqslant r(t)+\int_{a}^{t} f(s)(x(s))^{\ell} \mathrm{d} s .
$$

If the following inequality

$$
1-(\ell-1) \int_{a}^{t}(r(s))^{\ell-1} f(s) \mathrm{d} s>0,
$$

holds, then

$$
x(t) \leqslant \frac{r(t)}{\left(1-(\ell-1) \int_{a}^{t}(r(s))^{\ell-1} f(s) \mathrm{d} s\right)^{1 /(\ell-1)}} .
$$

